\title{
Application of "Primed" Mesenchymal Stromal Cells in Hematopoietic Stem Cell Transplantation: Current Status and Future Prospects
}

\author{
Vaijayanti P. Kale
}

Regenerative potential of mesenchymal stem/stromal cells (MSCs) has led to their application in various cellular therapies. Since in vivo these cells are present in very low numbers, they need expansion in culture to get clinically relevant numbers; however, such long-term ex vivo manipulation leads to loss of their regenerative capacity. Although use of naïve MSCs is still the most common approach used in various therapies, several strategies, both genetic and pharmacological, are being tried out to boost the regenerative capacity of in vitro expanded MSCs. Such manipulations are very commonly reported for regeneration of various tissues like bone, cartilage, kidney, pancreas, and others. Likewise, several efforts have been made to investigate priming of MSCs to enhance their immunoregulatory activity, but such efforts have not been made to the same extent for enhancing the efficacy of hematopoietic stem cell transplantation (HSCT). Development of such approaches for HSCT would not only be useful for enhancing the transplantation efficacy of cord blood cells, which are fewer in numbers, and aged HSCs, which could be functionally compromised, but also for genetically modified HSCs, which are likely to be both, fewer in number and functionally compromised. This review specifically deals with application of "primed" MSCs in the scenario of HSCT.

Keywords: primed, MSCs, HSCs, GvHD, engraftment, extracellular vesicles

\section{Introduction}

$\mathrm{M}$ ESENCHYMAL STEM/STROMAL CELLS (MSCs) are ubiquitously present in most tissues, and though their precise in vivo function has not been clearly elucidated, it is assumed that they support the regeneration of tissues in which they reside. In recent years the regenerative potential of exogenously provided MSCs for various tissue and cell types has been documented. This recognition opened a vast area of research in regenerative medicine and the literature got flooded with various reports describing their efficacy in both, experimental and clinical applications.

Initially the focus was on bone marrow-derived MSCs (BM-MSCs), but later on use of MSCs isolated from other sources like cord blood, cord tissue, placenta, adipose tissue, and so on became more prevalent, primarily because of the ease of the collection of these tissues and involvement of relatively fewer ethical issues. Since these cells are present in very low numbers in vivo, it becomes necessary to expand them in vitro before their application in regenerative medicine protocols. However, it was realized that such longterm culturing affects their phenotype [1], and perhaps also their functionality [2]. This recognition stimulated an intensive search for various strategies that can be applied to increase the regenerative capacity of in vitro expanded MSCs toward the target tissue.

One of the important aspects involved in the efficacious applications of MSCs for tissue regeneration is their homing to the target tissues after infusion. Most of the intravenously injected MSCs get entrapped into the microvasculature of the lungs, and therefore, very few MSCs actually reach the target tissue. Several approaches like direct administration in the affected tissues, genetic modification, cell surface engineering, priming, and others are being applied to improve their homing [3-7]. However, most of this work has been done for the tissues like bone, cartilage, pancreas, liver, kidney, brain, heart, and so on. [3,4], but not to the same extent for enhancing the efficacy of hematopoietic stem cell transplantation (HSCT).

Although, initially it was thought that the MSCs bring about regenerative changes via tissue-specific differentiation and integration, it was soon realized that they exert their therapeutic effects via their secretome [8]. Later it was also found that the composition of this secretome can be modulated by preconditioning the MSCs by hypoxia, cytokines,

Symbiosis Centre for Stem Cell Research, Symbiosis School of Biological Sciences, Symbiosis International University, Pune, India. 
growth factors, pharmacological agents, and the like or by simply growing them under three-dimensional culture conditions $[9,10]$.

Such modulation approaches are being extensively investigated for immunomodulation as well as for other regenerative therapies. However, in the area of HSCT most of such priming- or modulation-related work has been done with direct treatments of HSCs with pharmacological agents [11-14], by genetic manipulations [15] or with physical factors such as hypoxia [16-18]. However, direct manipulation of HSCs using pharmacological agents could have off target and/or unanticipated effects on their functionality [19], and therefore, priming of MSCs for boosting the engraftment capacity of the HSCs appears to be a safer approach and needs to be investigated more aggressively.

MSCs are being used in hematological applications for the treatment or prevention of graft-versus-host disease, support of hematopoiesis, or repair of tissue toxicities after hematopoietic cell transplantation [20]. Co-infusion of MSCs has been shown to boost the engraftment levels of transplanted HSCs [21-24], however, very few reports have documented the effect of co-infusion of "primed MSCs" on engraftment of donor HSCs. Using murine models it has been shown that co-infusion of HSCs with MSCs overexpressing CXCR-4 or SDF-1/HOXB4 improved post-transplant hematopoietic recovery $[25,26]$. Likewise, intra-marrow injection of beta-catenin-activated, but not naïve, MSCs was found to stimulate self-renewal of transplanted HSCs [27]. However, infusion of such genetically modified MSCs in human subjects could raise several safety issues, and therefore, ex vivo manipulation of HSCs with MSCs primed with pharmacological compounds would perhaps be a safer and more practicable approach.

This review specifically deals with the approaches that have been taken for priming of the MSCs with pharmacological compounds or various physical factors for improving the efficacy of HSCT in the context of HSC engraftment and control of graft versus host disease (GvHD). Development of such effective protocols would not only be useful for enhancing the efficacy of transplantation of cord blood cells, which are fewer in numbers, and aged HSCs, which are functionally compromised, but also for genetically modified HSCs, which are likely to be fewer in number and could also have compromised functionality.

\section{Priming of MSCs to Enhance Their Immunomodulatory Activities for Reducing GvHD}

HSCT is widely used to treat various malignant and nonmalignant conditions like hematological diseases, autoimmune diseases, primary immunodeficiency diseases, and inborn errors of metabolism [28]; however, GvHD is one of the major causes of post-transplant morbidity and mortality $[28,29]$. More than $50 \%$ of the transplanted patients do not respond to the standard first-line treatment of corticosteroids and these steroid-refractory patients generally show a higher mortality rate [30]. Since an established second-line treatment for steroid-refractory GvHD is not available, there is an urgent need for new therapies in these patients [31].

Due to their immunoregulatory properties, MSCs are being used for combating GvHD [32]. The mechanism involved in the immunoregulatory activity of MSCs has been extensively investigated [33] and has been shown to involve both, cell-cell interactions and secreted factors. Programmed death-1 receptor/programmed death-1 receptor ligand (PD1/PD-L1), B7 family immunoregulatory orphan ligand $\mathrm{H} 4$ (B7-H4), intercellular adhesion molecules of adhesion molecule family (ICAM), and vascular cell adhesion molecule (VCAM) [34-36] have so far been identified as some of the important molecules involved in contact-dependent immunosuppression by MSCs. In addition to these cell-cell interactions, MSCs secrete anti-inflammatory molecules including transforming growth factor- $\beta$ (TGF- $\beta$ ), hepatocyte growth factor (HGF), prostaglandin $\mathrm{E}_{2}\left(\mathrm{PGE}_{2}\right)$, indoleamine 2,3-dioxygenase (IDO), nitric oxide (NO), interluekin-6, TNF- $\alpha$-stimulated gene 6 protein (TSG-6) $[37,38]$ and so on that inhibit the proliferation and/or functions of $\mathrm{CD}^{+}{ }^{+} \mathrm{Th} 1$ and Th17 cells, $\mathrm{CD}^{+} \mathrm{T}$ cells, and natural killer cells.

Efforts are being done to increase this base level immunoregulatory activity of the MSCs. Kim et al. [39] showed that priming of MSCs with hypoxia and calcium ions boosts their immunoregulatory activity leading to better reduction in GvHD, when compared to the naïve MSCs. They attributed these beneficial effects of primed MSCs for upregulation of pololike kinase-1 (PLK1), zinc-finger protein-143, dehydrogenase/ reductase-3, and friend-of-GATA2. Recently, it was found that priming of MSCs with IFN- $\gamma$ increases their immunomodulatory properties [40]. Similar findings have also been reported by Kim et al. [41]. They showed that infusion of IFN- $\gamma$-primed MSCs, but not of naïve MSCs, reduced the GvHD symptoms in nonobese diabetic/severe combined immunodeficiency (NOD/SCID) mouse model. This effect was mediated via expression of IDO gene in MSCs via the IFN- $\gamma$-Janus kinase (JAK)-signal transducer and activator of transcription 1 (STAT1) pathway, and the infusion of IDO-overexpressing MSCs increased survival rate in an in vivo GvHD model, similar to infusion of IFN- $\gamma$-primed MSCs.

However, in this context, two reports need to be taken into consideration. Goedhart et al. [42] have demonstrated that though the treatment of MSCs with IFN-gamma increases their immunosuppressive activity, it significantly reduces their HSC-supportive ability. This study puts a question mark on the application of IFN-gamma-primed MSCs in the scenario of HSCT. Second, Li et al. [43] have shown that instead of immunosuppression, MSCs would "enhance" immune responses if proinflammatory cytokines were inadequate to elicit sufficient NO production in them. This study points toward the need of screening of the MSCs for their cytokine profile before their application.

Saparov et al. [44] have reviewed approaches in preconditioning human MSCs with hypoxia or cytokines for augmenting their capacity to regulate both innate and adaptive immune responses. Similar priming of MSCs by TOLL-like receptor 3 ligand has also been reported [45]. TLR3 ligand-treated MSCs released proinflammatory cytokines viz. interleukin (IL)-6 and IL-8 and also type I interferon in a biphasic manner. Sangiorgi et al. [46] investigated the modulation of the immune suppressive potential of BMMSC by different TLR ligands. They found that immunosuppressive capacity of MSCs was hampered by priming of TLR4 by lipopolysaccharide (LPS; possibly due to the activation of canonical NF- $\mathrm{KB}$ pathway), while TLR9 priming by $\mathrm{CpG}$ oligonucleotide enhanced their immune suppression, perhaps due to the activation of non-canonical NF- $\kappa \mathrm{B}$ 
pathway. These reports indicate that priming of MSCs with specific agents could boost their inherent immunomodulatory properties.

Saldaña et al. [47] found that priming of MSCs with soluble factors produced by both, pro-inflammatory (M1 type) or anti-inflammatory (M2 type) macrophages enhanced their immunomodulatory potential through increased $\mathrm{PGE}_{2}$ secretion. They identified the proinflammatory cytokine TNF- $\alpha$ as a priming factor for MSCs, and interestingly, antiinflammatory cytokine IL-10 potentiated the priming effect of TNF- $\alpha$. Showalter et al. [48] found that hypoxiaand serum-deprivation primed MSCs undergo glycolytic reprogramming, which homogenizes MSCs' metabolomic profile. In addition, they found that exosomes derived from these primed MSCs are packaged with numerous metabolites that have been directly associated with immunomodulation, including M2 macrophage polarization and regulatory $\mathrm{T}$ lymphocyte induction, suggesting that such primed-MSCs could have better therapeutic effects.

HSCT has been shown to improve skin blistering in pediatric patients with recessive dystrophic epidermolysis bullosa (RDEB), a genetic disorder resulting from loss-offunction mutations in COL7A1 as evidenced by absent or deficient type VII collagen protein (C7) in the epidermal basement membrane. MSCs present in the graft are thought to be partly responsible for this amelioration by their immunomodulatory and trophic effects and their ability to restore C7 protein in RDEB models [49]. Using a murine model Perdoni et al. [49] demonstrated that MSCs treated with exogenous TGF- $\beta(15 \mathrm{ng} / \mathrm{mL})$ and TNF $\alpha(30 \mathrm{ng} / \mathrm{mL})$ for $48 \mathrm{~h}$ induced an eightfold elevation in Col7al expression, an elevation in the secretion of $\mathrm{C} 7$ protein, and a fourfold increase in Tsg-6 expression. Tsg-6 protein is suggested to improve healing of wounds and immunosuppression.

Collectively, these reports show that many efforts have been made to investigate priming of MSCs to enhance their immunoregulatory activity in the context of GvHD.

\section{Priming of MSCs to Enhance HSC Engraftment}

Efficacy of HSCT primarily depends on the efficient homing, engraftment, proliferation, and multi-lineage differentiation of the donor HSCs. While several studies are available on ex vivo expansion of HSCs using MSCs as feeder layers, priming of MSCs to enhance homing and engraftment capacity of donor HSCs is not being investigated in great details. Liu et al. [50] showed that mechanopriming of hMSCs alters their secretome. They found that MSCs grown on the least stiff (elastic moduli $\sim 1 \mathrm{kPa}$ ) of polydimethylsiloxane (PDMS) substrata secreted high concentrations of key proteins that are known to be effective in ameliorating the effects of radiation. These MSCs increased hematopoiesis in an in vitro coculture model with hematopoietic stem and progenitor cells (HSPCs). On the other hand, MSCs cultured on PDMS of highest stiffness (elastic moduli $\sim 100 \mathrm{kPa}$ ) elevated expression of CD123 ${ }^{+}$HSPCs, suggesting myeloid differentiation. Systemic administration of mechano-primed MSCs resulted in improved mouse survival (irradiated NOD/SCID mice) and resulted in a total recovery of all hematopoietic lineages, when compared with MSC expanded on stiffer materials. These secreted factors included IL-6, IL-8, bone morphogenetic protein 2 (BMP2), epidermal growth factor (EGF), fibroblast growth factor 1 (FGF1), regulated on activation, normal $\mathrm{T}$ cell expressed and secreted (RANTES), vascular endothelial growth factor A (VEGF-A), and angiopoietin-1 (ANG-1).

Since BM is a hypoxic tissue, several studies employed hypoxia to modulate MSCs as feeder layers for the cultures of HSCs. Most effects of hypoxia are mediated by the action of HIF1 $\alpha$, and hence, Kiani et al. [51] used HIF1 $\alpha$ overexpressing MSCs to expand cord blood (CB)-derived CD34 $4^{+}$ HSCs. They found that overexpression of HIF1 $\alpha$ in BMderived MSCs improves their HSC-supportive functions and leads to enhanced colony formation ability of the cocultured HSCs. This effect was correlated with the higher levels of SCF produced by the HIF1 $\alpha$-modified MSCs. In these studies the engraftment ability of cultured HSCs was not investigated. Coculture of murine HSCs with MSCs treated with hypoxia (1\% Oxygen) or cobalt chloride (Hypoxia mimetic) was shown to boost their engraftment ability [52]. Likewise, Zhao et al. [53] showed that coculture of cord blood-derived CD34 ${ }^{+}$HSCs with hypoxia-treated Wharton Jelly-derived MSCs improved their in vitro functionality. Sharma et al. [54] showed that three-dimensional hydrogelbased cultures of placenta-derived MSCs expand human BM-derived CD34 ${ }^{+}$HSCs and boost their engraftment ability owing to the formation of hypoxia gradient. Importantly, formation of hypoxia gradient, but not complete hypoxia, was necessary to achieve such salutary effects on the HSCs.

Genetic manipulation of feeder layers has been shown to impart better yield of HSCs. Wenk et al. [55] showed that 5Azacitidine (5Aza) treatment of MSCs isolated from both, normal and myelodysplastic syndromes marrows resulted in their epigenetic modulation and these 5Aza-modulated MSCs supported the growth of normal HSCs over leukemic HSCs. However, in this study only colony formation assay was used as a read out and in vivo transplantation studies were not done. Nonetheless, this approach could be useful in expanding HSCs collected for auto HSCT. This way the likelihood of leukemic cells, if present in the harvested samples, getting expanded would become negligible. This possibility needs to be formally examined.

Using an RNA sequencing screen, Nakahara et al. [56] found that expression of five transcription factors, namely, Klf7, Ostf1, Xbp1, Irf3, and Irf7, in cultured BM-MSCs restored HSC niche function in them. They further showed that these modified MSCs secreted higher amounts of niche factors and coculture of HSCs with these "revitalized" MSCs, but not with naïve MSCs, showed higher repopulation capacity. Using single-cell mass cytometry, Severe et al. [57] defined 28 subsets of bone marrow stromal cells based on protein expression. They showed that irradiationbased conditioning of mice resulted in loss of most of these subsets, except $\mathrm{CD}_{73}{ }^{+} \mathrm{NGFR}^{+}$stromal cells. This was the only radio-resistant population, which played a crucial part in engraftment and acute hematopoietic recovery. This effect could be correlated with a significantly higher expression of various cytokines, especially of G-CSF, and other factors implicated in hematopoietic niche function. These data suggest that $\mathrm{CD}^{+} 3^{+} \mathrm{NGFR}^{+}$MSCs could either be enriched by sorting from the bulk MSC cultures, or the cultured MSCs could be modulated by genetic or pharmacological means to enhance the expression of these molecules. Such 
selected or primed MSCs could be used to expand functional HSCs in vitro to achieve early recovery of post-transplant hematopoiesis.

\section{Role of Extracellular Vesicles in HSCT}

Recent literature has clearly shown that extracellular vesicles (EVs), both, micro-vesicles (MVs) and exosomes, form an important pathway of intercellular communication between MSCs and HSCs [58], as these vesicles are enriched in various types of macromolecules such as proteins, metabolites, messenger RNA (mRNA), microRNA (miRNA), and others. Therefore, an intercellular exchange of these vesicles affects the fate of the target cells [59].

Therapeutic effects of MSC-derived EVs on various tissues [60-64] and on expansion and engraftment of HSCs have been documented [65,66]. However, most of these studies have been done with EVs isolated from naïve MSCs. Not much attention has been given on the effect of priming of MSCs on the macromolecular composition of their EVs and the consequent effect on the target cells. We have previously shown that signaling mechanisms prevailing in the MSCs affect the macromolecular composition of the EVs secreted by them and alter the effect on HSCs cocultured with them [67]. We specifically demonstrated that activation of AKT in the MSCs leads to secretion of MVs having detrimental effects on the engraftment ability of the HSCs cocultured with them. Therefore, identification of signaling gamut present in the MSCs used for the collection of therapeutic EVs may be helpful.

MSC-derived EVs have been shown to prompt a loss of HSPC quiescence with concomitant expansion of murine myeloid progenitors via TOLL-like receptor engagement [68]. Intravenous infusion of MSC (both, human and mouse)-derived EVs was shown to reverse irradiationinduced damage to murine marrow hematopoietic cells [69]. Interestingly, in this study the investigators found that though both, MVs and exosomes were effective independently, a combined infusion of MVs and exosomes was found to give better results. On the other hand, we found that MVs isolated from young MSCs rejuvenated aged HSCs and boosted their engraftment, whereas exosomes exerted a negative effect [70]. Aged exosomes affected the engraftment ability of young HSCs due to the aging-mediated partitioning of MiR 17 and MiR34a, which downregulate autophagy-inducing genes. This situation could be rescued by pharmacological inhibition of AKT in the aged MSCs, thereby showing that it is possible to rejuvenate aged MSCs by pharmacological means. Liu et al. [71] have shown that human induced pluripotent stem cell-derived EVs reduce senescence of MSCs by reducing their oxidative stress. We have also shown that treatment of MSCs with NO donors boosted their HSC-supportive properties due to partitioning of mRNAs encoding HSC-supportive genes like Jagged-1 and VEGF-A into their MVs, and coculturing of HSCs with such NO-primed MSCs or their MVs resulted in significantly improved engraftment of both adult and neonatal HSCs [19].

\section{Summary and Future Prospects}

HSCT is the oldest and the most successful stem cell therapy and has become a standard care treatment for sev- eral malignant and nonmalignant conditions. Despite years of success, certain problems like GvHD and graft failure due to low HSC number in cord blood units and reduced functionality of HSCs collected from aged donors still persist. Owing to their immunomodulatory activity, MSCs and their EVs are being used in preclinical and clinical studies to reduce the morbidity associated with GvHD. A recent trend of using primed MSCs to boost their immunosuppressive ability to get better results in controlling GvHD is very encouraging, however, the effect of such primed MSCs on HSCs also needs to be evaluated beforehand.

Recent studies show that EVs possess equivalent immunomodulatory activity as that of their parental MSCs. EVs have distinct advantages over MSCs: they can be easily cryopreserved and, thus, form a "ready-to-use" biologic; owing to their nanoscale size they can easily circulate in the microvasculature and even pass blood-brain barrier; and being acellular entities they cannot self-replicate, thereby avoiding the danger of tumor formation. It is however, important to modulate the macromolecular composition of EVs by appropriately priming the parent MSCs.

In addition to their immunoregulatory activities, MSCs also possess HSC-supportive activity and therefore, are being used to improve the engraftment ability of donor HSCs. However, very few studies are done by using primed MSCs or their EVs in this regard. Such efforts are needed to gain advantage in terms of increased engraftment of donor HSCs. This could not only increase the donor cohort, but may also reduce the expenses incurred in post-transplant hospital care. With the advent of precise gene therapy protocols like crisper/cas9, transplantation with genetically modified HSCs would soon become a reality for several genetic diseases. These genetically modified HSCs are fewer in number and hence need ex vivo expansion. In this context efforts need to be done to search for appropriately primed MSCs to expand these gene-modified HSCs having superior engraftment capacity.

\section{Acknowledgment}

I wish to thank the anonymous reviewer for the excellent critique on my article.

\section{Author Disclosure Statement}

The author declares no conflict of interest.

\section{Funding Information}

This work was supported by intra-mural grants by Symbiosis International University (SIU).

\section{References}

1. Bara JJ, RG Richards, M Alini and MJ Stoddart. (2014). Concise review: bone marrow-derived mesenchymal stem cells change phenotype following in vitro culture: implications for basic research and the clinic. Stem Cells 32: 1713-1723.

2. Abbuehl JP, Z Tatarova, W Held and J Huelsken. (2017). Long-term engraftment of primary bone marrow stromal cells repairs niche damage and improves hematopoietic stem cell transplantation. Cell Stem Cell 21:241255 . 
3. Yu SP, Z Wei and L Wei. (2013). Preconditioning strategy in stem cell transplantation therapy. Transl Stroke Res 4: $76-88$.

4. Schäfer R, G Spohn and PC Baer. (2016). Mesenchymal stem/stromal cells in regenerative medicine: can preconditioning strategies improve therapeutic efficacy? Transfus Med Hemother 43:256-267.

5. Hu C and L Li. (2018). Preconditioning influences mesenchymal stem cell properties in vitro and in vivo. J Cell Mol Med 22:1428-1442.

6. Ullah M, DD Liu and AS Thakor. (2019). Mesenchymal stromal cell homing: mechanisms and strategies for improvement. iScience 15:421-438.

7. Yin JQ, J Zhu and JA Ankrum. (2019). Manufacturing of primed mesenchymal stromal cells for therapy. Nat Biomed Eng 3:90-104.

8. Vizoso FJ, N Eiro, S Cid, J Schneider and R PerezFernandez. (2017). Mesenchymal stem cell secretome: toward cell-free therapeutic strategies in regenerative medicine. Int J Mol Sci 18:1852-1876.

9. Ferreira JR, GQ Teixeira, SG Santos, MA Barbosa, G Almeida-Porada and RM Gonçalves. (2018). Mesenchymal stromal cell secretome: influencing therapeutic potential by cellular pre-conditioning. Front Immunol 9:2837-2854.

10. Cunningham CJ, E Redondo-Castro and SM Allan. (2018). The therapeutic potential of the mesenchymal stem cell secretome in ischaemic stroke. J Cereb Blood Flow Metab 38:1276-1292.

11. Ko KH, T Holmes, P Palladinetti, E Song, R Nordon, TA O'Brien and A Dolnikov. (2011). GSK-3 $\beta$ inhibition promotes engraftment of ex vivo-expanded hematopoietic stem cells and modulates gene expression. Stem Cells 29: 108-118.

12. Lam BS, C Cunningham and GB Adams. (2011). Pharmacologic modulation of the calcium-sensing receptor enhances hematopoietic stem cell lodgment in the adult bone marrow. Blood 117:1167-1175.

13. Itkin T, A Ludin, B Gradus, S Gur-Cohen, A Kalinkovich, A Schajnovitz, Y Ovadya, O Kollet, J Canaani, et al. (2012). FGF-2 expands murine hematopoietic stem and progenitor cells via proliferation of stromal cells, c-Kit activation, and CXCL12 down-regulation. Blood 120: 1843-1855.

14. Hu L, H Cheng, Y Gao, M Shi, Y Liu, Z Hu, J Xu, L Qiu, W Yuan, et al. (2014). Antioxidant $N$-acetyl-L-cysteine increases engraftment of human hematopoietic stem cells in immune-deficient mice Blood 124:e45-e48.

15. Takizawa H, C Kubo-Akashi, I Nobuhisa, SM Kwon, M Iseki, T Taga, K Takatsu and S Takaki. (2006). Enhanced engraftment of hematopoietic stem/progenitor cells by the transient inhibition of an adaptor protein, Lnk. Blood 107: 2968-2975.

16. Simsek T, F Kocabas, J Zheng, RJ Deberardinis, AI Mahmoud, EN Olson, JW Schneider, CC Zhang and HA Sadek. (2010). The distinct metabolic profile of hematopoietic stem cells reflects their location in a hypoxic niche. Cell Stem Cell 7:380-390.

17. Takubo K, N Goda, W Yamada, H Iriuchishima, E Ikeda, Y Kubota, H Shima, RS Johnson, A Hirao, M Suematsu and T Suda. (2010). Regulation of the HIF-1a level is essential for hematopoietic stem cells. Cell Stem Cell 7:391-402.

18. Pollard PJ and KR. Kranc. (2010). Hypoxia signaling in hematopoietic stem cells: a double-edged sword. Cell Stem Cell 7:276-278.
19. Jalnapurkar S, RD Moirangthem, S Singh, L Limaye and V Kale. (2019). Microvesicles secreted by nitric oxide-primed mesenchymal stromal cells boost the engraftment potential of hematopoietic stem cells. Stem Cells 37:128-138.

20. De Becker A and I Van Riet. (2015). Mesenchymal stromal cell therapy in hematology: from laboratory to clinic and back again. Stem Cells Dev 24:1713-1729.

21. Battiwalla M and P Hematti. (2009). Mesenchymal stem cells in hematopoietic stem cell transplantation. Cytotherapy 11:503-515.

22. Carrancio S, C Romo, T Ramos, N Lopez-Holgado, S Muntion, HJ Prins, AC Martens, JG Briñón, JF San Miguel, MC Del Cañizo and F Sanchez-Guijo. (2013). Effects of MSC coadministration and route of delivery on cord blood hematopoietic stem cell engraftment. Cell Transplant 22: 1171-1183.

23. Fernández-García M, RM Yañez, R Sánchez-Domínguez, M Hernando-Rodriguez, M Peces-Barba, G Herrera, JE O'Connor, JC Segovia, JA Bueren and ML Lamana. (2015). Mesenchymal stromal cells enhance the engraftment of hematopoietic stem cells in an autologous mouse transplantation model. Stem Cell Res Ther 6:165-178.

24. Zhao K and Q Liu. (2016). The clinical application of mesenchymal stromal cells in hematopoietic stem cell transplantation. J Hematol Oncol 9:46-55.

25. Chen T, P Zhang, W Fan, F Qian, L Pei, S Xu, Z Zou, B Ni and Y Zhang. (2014). Co-transplantation with mesenchymal stem cells expressing a SDF-1/HOXB4 fusion protein markedly improves hematopoietic stem cell engraftment and hematogenesis in irradiated mice. Am J Transl Res 6: 691-702.

26. Chen W, M Li, G Su, Y Zang, Z Yan, H Cheng, B Pan, J Cao, Q Wu, et al. (2015). Co-transplantation of hematopoietic stem cells and Cxcr4 gene-transduced mesenchymal stem cells promotes hematopoiesis. Cell Biochem Biophys 71:1579-1587.

27. Ahn JY, G Park, JS Shim, JW Lee and IH Oh. (2010). Intramarrow injection of $\beta$-catenin-activated, but not naïve mesenchymal stromal cells stimulates self-renewal of hematopoietic stem cells in bone marrow. Exp Mol Med 42: 122-131.

28. Ringdén O, M Uzunel, I Rasmusson, M Remberger, B Sundberg, H Lönnies, HU Marschall, A Dlugosz, A Szakos, et al. (2006). Mesenchymal stem cells for treatment of therapy-resistant graft-versus-host disease. Transplantation 81:1390-1397.

29. Qian L, Z Wu and J Shen. (2013). Advances in the treatment of acute graft-versus-host disease. J Cell Mol Med 17: 966-975.

30. Bürgler D, M Medinger, J Passweg, A Fischmann and C Bucher. (2014). Intra-arterial catheter guided steroid administration for the treatment of steroid-refractory intestinal GvHD. Leuk Res 38:184-187.

31. Medinger M, A Tichelli, C Bucher, J Halter, S Dirnhofer, A Rovo, J Passweg and A Tzankov. (2013). GVHD after allogeneic haematopoietic SCT for AML: angiogenesis, vascular endothelial growth factor and VEGF receptor expression in the BM. Bone Marrow Transplant 48:715-721.

32. Kim EJ, N Kim and SG Cho. (2013). The potential use of mesenchymal stem cells in hematopoietic stem cell transplantation. Exp Mol Med 45:e2.

33. Weiss ARR and MH Dahlke. (2019). Immunomodulation by mesenchymal stem cells (MSCs): mechanisms of action of living, apoptotic, and dead MSCs. Front Immunol 10:1191. 
34. Augello A, R Tasso, SM Negrini, A Amateis, F Indiveri, R Cancedda and P Giuseppina. (2005). Bone marrow mesenchymal progenitor cells inhibit lymphocyte proliferation by activation of the programmed death 1 pathway. Eur $\mathbf{J}$ Immunol 35:1482-1490.

35. Xue Q, XY Luan, YZ Gu, HY Wu, GB Zhang, GH Yu, HT Zhu, M Wang, W Dong, YJ Geng and XG Zhang. (2010). The negative cosignaling molecule B7-H4 is expressed by human bone marrow-derived mesenchymal stem cells and mediates its T-cell modulatory activity. Stem Cells Dev 19: 27-38.

36. Ren G, X Zhao, L Zhang, J Zhang, A L'Huillier, W Ling, AI Roberts, AD Le, S Shi, C Shao and Y Shi. (2010). Inflammatory cytokine induced intercellular adhesion molecule-1 and vascular cell adhesion molecule-1 in mesenchymal stem cells are critical for immunosuppression. $\mathbf{J}$ Immunol 184:2321-2328.

37. Zhou Y, Y Yamamoto, Z Xiao and O Takahiro. (2019). The immunomodulatory functions of mesenchymal stromal/stem cells mediated via paracrine activity. J Clin Med 8:1025.

38. Choi H, RH Lee, N Bazhanov, JY Oh and DJ, Prockop. (2011). Anti-inflammatory protein TSG-6 secreted by activated MSCs attenuates zymosan-induced mouse peritonitis by decreasing TLR2/NF- $\kappa \mathrm{B}$ signaling in resident macrophages. Blood 118:330-338.

39. Kim Y, HJ Jin, J Heo, H Ju, HY Lee, S Kim, S Lee, J Lim, SY Jeong, et al. (2018). Small hypoxia-primed mesenchymal stem cells attenuate graft-versus-host disease. Leukemia 32:2672-2684.

40. Sivanathan KN, S Gronthos, D Rojas-Canales, B Thierry and PT Coates. (2014). Interferon-gamma modification of mesenchymal stem cells: implications of autologous and allogeneic mesenchymal stem cell therapy in allotransplantation. Stem Cell Rev 10:351-375.

41. Kim DS, IK Jang, MW Lee, YJ Ko, DH Lee, JW Lee, KW Sung, HH Koo and KH Yoo. (2018). Enhanced immunosuppressive properties of human mesenchymal stem cells primed by interferon- $\gamma$. EBioMedicine 28:261-273.

42. Goedhart M, AS Cornelissen, C Kuijk, S Geerman, M Kleijer, JD van Buul, S Huveneers, MHGP Raaijmakers, HA Young, et al. (2018). Interferon-gamma impairs maintenance and alters hematopoietic support of bone marrow mesenchymal stromal cells. Stem Cells Dev 27:579-589.

43. Li W, G Ren, Y Huang, J Su, Y Han, J Li, X Chen, K Cao, Q Chen, et al. (2012). Mesenchymal stem cells: a doubleedged sword in regulating immune responses Cell Death Differ 19:1505-1513.

44. Saparov A, V Ogay, T Nurgozhin, M Jumabay and WC Chen. (2016). Preconditioning of human mesenchymal stem cells to enhance their regulation of the immune response. Stem Cells Int 2016:3924858.

45. Dumitru CA, H Hemeda, M Jakob, S Lang and S Brandau. (2014). Stimulation of mesenchymal stromal cells (MSCs) via TLR3 reveals a novel mechanism of autocrine priming. FASEB J 28:3856-3866.

46. Sangiorgi BB, V Leão, JL dos Santos Schiavinato, MD Orellana, MA Zago, DT Covas, RA Panepucci and EM Rego. (2013). TLR9 priming promotes proliferation of mesenchymal stem cells and restores the immunosuppressive activity impaired by TLR 4 priming: potential involvement of non-canonical NF- $\kappa \mathrm{B}$ signaling. Blood 22: 2458.

47. Saldaña L, F Bensiamar, G Vallés, FJ Mancebo, E García-Rey and N Vilaboa. (2019). Immunoregulatory potential of mes- enchymal stem cells following activation by macrophagederived soluble factors. Stem Cell Res Ther 10:58-73.

48. Showalter MR, B Wancewicz, O Fiehn, JA Archard, S Clayton, J Wagner, P Deng, J Halmai, KD Fink, et al. (2019). Primed mesenchymal stem cells package exosomes with metabolites associated with immunomodulation. Biochem Biophys Res Commun 512:729-735.

49. Perdoni C, JA McGrath and J Tolar. (2014). Preconditioning of mesenchymal stem cells for improved transplantation efficacy in recessive dystrophic epidermolysis bullosa. Stem Cell Res Ther 5:121-133.

50. Liu FD, K Tam, N Pishesha, Z Poon and KJ Van Vliet. (2018). Improving hematopoietic recovery through modeling and modulation of the mesenchymal stromal cell secretome. Stem Cell Res Ther 9:268-281.

51. Kiani AA, J Abdi, R Halabian, MH Roudkenar, N Amirizadeh, M Soleiman Soltanpour and A Kazemi. (2014). Over expression of HIF- $1 \alpha$ in human mesenchymal stem cells increases their supportive functions for hematopoietic stem cells in an experimental coculture model. Hematology 19:85-98.

52. Moirangthem RD, S Singh, A Adsul, S Jalnapurkar, L Limaye and VP Kale. (2015). Hypoxic niche-mediated regeneration of hematopoiesis in the engraftment window is dominantly affected by oxygen tension in the milieu. Stem Cells Dev 24:2423-2436.

53. Zhao D, L Liu, Q Chen, F Wang, Q Li, Q Zeng, J Huang, M Luo, W Li, Y Zheng and T Liu. (2018). Hypoxia with Wharton's jelly mesenchymal stem cell coculture maintains stemness of umbilical cord blood-derived CD34 ${ }^{+}$cells. Stem Cell Res Ther 9:158-169.

54. Sharma MB, LS Limaye and VP. Kale. (2012). Mimicking the functional hematopoietic stem cell niche in vitro: recapitulation of marrow physiology by hydrogel-based three-dimensional cultures of mesenchymal stromal cells. Haematologica 97:651-660.

55. Wenk C, AK Garz, S Grath, C Huberle, D Witham, M Weickert, R Malinverni, J Niggemeyer, M Kyncl, et al. (2018). Direct modulation of the bone marrow mesenchymal stromal cell compartment by azacitidine enhances healthy hematopoiesis. Blood Adv 2:3447-3461.

56. Nakahara F, DK Borger, Q Wei, S Pinho, M Maryanovich, AH Zahalka, M Suzuki, CD Cruz, Z Wang, et al. (2019). Engineering a haematopoietic stem cell niche by revitalizing mesenchymal stromal cells. Nat Cell Biol 21:560-567.

57. Severe N, NM Karabacak, K Gustafsson, N Baryawno, G Courties, Y Kfoury, KD Kokkaliaris, C Rhee, D Lee, et al. (2019). Stress-induced changes in bone marrow stromal cell populations revealed through single-cell protein expression mapping. Cell Stem Cell 25:1-14.

58. Laurenzana I, D Lamorte, S Trino, L De Luca, C Ambrosino, P Zoppoli, V Ruggieri, L Del Vecchio, P Musto, A Caivano and G Falco. (2018). Extracellular vesicles: a new prospective in crosstalk between microenvironment and stem cells in hematological malignancies. Stem Cells Int 2018:9863194.

59. Raposo G and W Stoorvogel. (2013). Extracellular vesicles: exosomes, microvesicles, and friends. J Cell Biol 200:373383.

60. Bruno S, S Porta and B Bussolati. (2016). Extracellular vesicles in renal tissue damage and regeneration. Eur $\mathbf{J}$ Pharamacol 790:83-91.

61. Lai RC, F Arslan, MM Lee, NS Sze, A Choo, TS Chen, M Salto-Tellez, L Timmers, CN Lee, et al. (2010). Exosome 
secreted by MSC reduces myocardial ischemia/reperfusion injury. Stem Cell Res 4:214-222.

62. Xin H, Y Li, B Buller, M Katakowski, Y Zhang, X Wang, X Shang, ZG Zhang and M Chopp. (2012). Exosomemediated transfer of miR-133b from multipotent mesenchymal stromal cells to neural cells contributes to neurite outgrowth. Stem Cells 30:1556-1564.

63. Keshtkar S, N Azarpira and MH Ghahremani. (2018). Mesenchymal stem cell-derived extracellular vesicles: novel frontiers in regenerative medicine. Stem Cell Res Ther 9:63-72.

64. Baek G, H Choi, Y Kim, HC Lee and C Choi. (2019). Mesenchymal stem cell-derived extracellular vesicles as therapeutics and as a drug delivery platform. Stem Cells Transl Med 8:880-886.

65. Xie H, L Sun, L Zhang, T Liu, L Chen, A Zhao, Q Lei, F Gao, P Zou, et al. (2016). Mesenchymal stem cell-derived microvesicles support ex vivo expansion of cord bloodderived CD34 ${ }^{+}$cells. Stem Cell Int 2016:6493241.

66. De Luca L, S Trino, I Laurenzana, D Lamorte, A Caivano, L Del Vecchio and P Musto. (2017). Mesenchymal stem cell derived extracellular vesicles: a role in hematopoietic transplantation? Int J Mol Sci 18:1022-1039.

67. Singh S, RD Moirangthem, A Vaidya, S Jalnapurkar, L Limaye and V Kale. (2016). AKT signaling prevailing in mesenchymal stromal cells modulates the functionality of hematopoietic stem cells via intercellular communication. Stem Cells 34:2354-2367.

68. Goloviznina NA, SC Verghese, YM Yoon, O Taratula, DL Marks and P Kurre. (2016). Mesenchymal stromal cell derived extracellular vesicles promote myeloid biased multipotent hematopoietic progenitor expansion via tolllike receptor engagement. J Biol Chem 291:24607-24617.

69. Wen S, M Dooner, Y Cheng, E Papa, M Del Tatto, M Pereira, Y Deng, L Goldberg, J Aliotta, et al. (2016). Mesenchymal stromal cell derived extracellular vesicles rescue radiation damage to murine marrow hematopoietic cells. Leukemia 30:2221-2231.

70. Kulkarni R, M Bajaj, S Ghode, S Jalnapurkar, L Limaye and V Kale. (2017). Intercellular transfer of microvesicles from young mesenchymal stromal cells rejuvenates aged murine hematopoietic stem cells. Stem Cells 36:420-433.

71. Liu S, V Mahairaki, H Bai, Z Ding, J Li, KW Witwer and L Cheng. (2019). Highly purified human extracellular vesicles produced by stem cells alleviate aging cellular phenotypes of senescent human cells. Stem Cells 36:420-433.

Address correspondence to: Dr. Vaijayanti P. Kale Symbiosis Centre for Stem Cell Research (SCSCR) Symbiosis School of Biological Sciences Symbiosis Knowledge Park, Lavale Pune 412112 India

E-mail: vaijayanti.kale@ssbs.edu.in vaijayanti.kale@gmail.com

Received for publication July 16, 2019 Accepted after revision September 26, 2019

Prepublished on Liebert Instant Online September 27, 2019 